this purpose as ergot is. Schwab, too, confirms the opinion I have long held, that ergot does not originate uterine action.

\section{A CASE OF SUPPURATIVE OTITIS MEDIA COMPLICATED WITH CEREBELLAR} \section{ABSCESS. ${ }^{1}$}

By H. SECKER WALKER, F.R.C.S.,

Assistant Ophthalmic and Aural Surgeon, Leeds General Infirmary ;

Lecturer on Ophthalmology and Otology at the Yorkshire
College, Victoria University.

ON November 22nd, 1895, I saw, in consultation with Mr. J. W. Hutton, C. J., aged i4. He had had right otorrhœa for some years with a polypus in this ear. I had removed the polypus two years earlier, and the otorrhœa ceased. Four months before the date on which I saw hir again he was attacked by pain in the left ear, which was followed by a purulent discharge, and lately this had been tinged with blood; the pain had returned and spread over the head and become constant, being located chiefly in the occipital region. The boy looked pale and had wasted rapidly. Five weeks earlier he had several attacks of shivering. He had vomited occasionally in the morning, constipation had been marked, and there had been some delirium at times.

On examination of the left ear a polypus was seen deep in the meatus, the discharge was very offensive, the mastoid region looked healthy, but there was tenderness on deep pressure. He was very deaf in both ears. The cervical glands were enlarged, and one or two superficial ones in front of the neck had ulcerated. The temperature had been between $99^{\circ}$ and $100^{\circ}$, the pulse from 110 to 130 . There was no optic neuritis, no paralysis, no spasm of any of the ocular or facial muscles. The grasp of the hands was weak, but not markedly more on one side than the other. The knee-jerk was increased on the left, normal on the right side. When spoken to he answered intelligently, and his answers were not delayed. In the presence of the above symptoms there could be no doubt about the condition of the mastoid process, but I did not then feel justified in diagnosing intracranial disease, as most of the above symptoms may be present in a simple case of disease of the mastoid cells.

The following operation was performed :

\section{First Operation.}

A curved incision, $2 \frac{1}{2}$ inches long, immediately behind the attachment of the ear was made down to the bune, the cartilaginous meatus stripped from its bony attachment and the ear turned forwards, the periosteum was pushed backwards, so that a considerable area of the temporal bone, including the external auditory meatus and the greater part of the mastoid process, was exposed together with the surface land marks which are of such great assistance in this operation. Immediately behind and slightly above the centre of the external auditory meatus lies the mastoid slightly above the centre of the external auditory meatus lies the mastoid easily broke this down and entered the antrum, which was filled with yellowish putty-like stuff with a very offensive odour. After clearing out this the posterior wall of the meatus and the external wall of the attic were chiselled away, and the carious ossicles and remains of the membrana tympani and polypus in the middle ear removed so that the middle ear and pani and polypus in the midale ear removed so that the middle ear and antrum pormed one cavity partly divided into two by a transverse ridge of bohewhis in me chiselled away, otherw secthe facial nervo will be divided, resultin in more or less permanent facial paralysis on this side. This acciden will be avoided by not cutting away bone below the upper third of the

The roof and posterior wall of the antrum were found carious and perforated so they were removed, exposing the dura mater lying against the temporo-sphenoidal convolutions and the lateral lobe of the cerebellum. The dura mater in each of these situations was firm, and, as I thought, probably limited the disease. After well washing out the cavity it was packed with iodoform gauze, and the superficial glandular abscess in the ower part of the neck was scraped.

For the next ten days the boy did well; he became brighter, the headache and vomiting ceased, the temperature fell a little, becoming subnormal, the pulse averaged i12, and he was only troubled by obstinate constipation. On the eleventh and twelfth days vomiting and headache again occurred; there was still no optic neuritis. The condition of the arm muscles and knee reflexes remained the same, but the boy was more prostrate. These symptoms continued; it appeared as if there must be some intracranial disease, and the symptoms pointed to cerebral or cerebellar abscess. From the amount of disease in the posterior and lower part of the Bead before the Leeds and West Riding Medico-Chirurgical Society. mastoid process lying near the lateral lobe of the cerebellum and the occipital headache, I thought this was the more likely place in the absence of any guiding symptoms. Dr. Churton and $\mathrm{Mr}$. Teale, who kindly saw the case, agreed with me as to the probability of intracranial abscess, and were also in favour of the cerebellum being the seat of the disease.

SECOND OPERATION.

The opening into the posterior fossa of the skull made at the first operation was enlarged, the dura mater reflected, and a silver drain tube passed into the cerebellum for an inch. On the second trial about 3 iij of very offensive pus welled slowly out of the tube. An unsatisfactory aft ind and lion.

The patient improved and the headache ceased, but the vomiting still occurred occasionally. Two days later the symptoms all reappeared; the tube was pushed in a little further, more pus came away, and again an attempt was made to wash out the cavity.

The condition of the boy now underwent much improvement, and next morning he was sitting up in bed calling for his breakfast, and much interested in the Yorkshire Post. For three weeks the improvement was maintained. On December 3 oth he had several attacks of vomiting; the wound looked well and there was practically no discharge from the tube. Thinking that the presence of the drainage tube, acting as a foreign body, might be causing the vomiting, I removed it. For a fortnight again he did very well, and then all the old symptoms returned with redoubled force; in a few days he changed from being a comparatively healthy-looking boy to a mere skeleton.

THIRD OPERATION.

On January $3^{\text {Ist }}$ I again opened the cerebellum, and this time a little further back. Pus was found without difficulty, and two decalcified bone tubes were introduced and carbolic lotion syringed gently through them.

From this time the boy made a rapid and complete recovery, the only drawback being that now for the first time with all this disease and surgical interference he developed an attack of double optic neuritis, which, however, disappeared in a few weeks without permanently damaging the vision.

The patient now has a cavity of considerable size in the left mastoid process, lined throughout with epidermis, and communicating with the external air by an opening behind the ear and by the external auditory meatus.

\section{REMARKS}

Recovery from cerebellar abscess is exceptional. As far as I have been able to learn there are only ro previous cases on record. The difficulties in diagnosis, the absence of any special localising symptoms, or even in some cases of any symptoms at all, and the liability to a sudden fatal termination of the case before treatment can be carried out, are probably among the chief reasons why so few cases recover.

In reporting a successful case of cerebellar abscess, Dr. Acland and Mr. Ballance ${ }^{2}$ have drawn attention to the occasional presence of certain paralytic symptoms which may be of great service in localising a cerebellar abscess, namely, muscular weakness of the limbs of the same side as the ear disease, affecting chiefly the arm, conjugate deviation of the eyes to the opposite side from weakness of the muscles, which draw the eye to the same side, and increased knee-jerk on the same side as the otorrhœa. That these symptoms have been present in some previously recorded and unsuccessful cases of cerebellar abscess a study of this interesting paper will prove, but their localising value had evidently not been understood; and, indeed, in one case the symptoms actually led the surgeon into the error of operating on the opposite and healthy side of the brain.

2 St. Thomas's Hospital Reports, vol. xxiii.

Medical Students at Austrian Universities.-The following figures show the number of students at each of the universities of Austria during the current semester ;-Vienna, 6,104; Graz, 1667; Prague (German), 1,391 ; Prague (Czech), 2,787 ; Innsbruck, I004; Lemberg, 1,556 ; Cracow, I, 296 ; Czernowitz, 39I. The total number of students belonging to the medical faculties of all these universities combined is 4,728 , which is rather less than in the corresponding period of last year. 